\title{
FINITE ORDER SOLUTIONS OF NONHOMOGENEOUS LINEAR DIFFERENTIAL EQUATIONS
}

\author{
Gary G. Gundersen and Enid M. Steinbart \\ University of New Orleans, Department of Mathematics \\ New Orleans, Louisiana 70148, U.S.A.
}

\begin{abstract}
We give conditions on the coefficients of equation (1.1) below which guarantee that every solution of (1.1) has infinite order. We also show that if the coefficients of (1.1) satisfy certain growth conditions, then any finite order solution of (1.1) satisfies certain corresponding growth conditions. Several examples are given to illustrate the results.
\end{abstract}

\section{Introduction}

In this paper we consider the nonhomogeneous linear differential equation

$$
f^{(n)}+A_{n-1}(z) f^{(n-1)}+\cdots+A_{1}(z) f^{\prime}+A_{0}(z) f=H(z)
$$

where $A_{0}(z), A_{1}(z), \cdots, A_{n-1}(z)$, and $H(z)$ are entire functions, $H(z) \not \equiv 0$, and $n \geq 1$. It is well known that every solution $f$ of (1.1) is entire.

In (1.1), if $p$ is the largest integer such that $A_{p}(z)$ is transcendental, then [6] there can exist at most $p$ linearly independent finite order solutions of the corresponding homogeneous equation

$$
f^{(n)}+A_{n-1}(z) f^{(n-1)}+\cdots+A_{1}(z) f^{\prime}+A_{0}(z) f=0 .
$$

Thus it can be deduced that "most" of the solutions of (1.1) with at least one $A_{k}(z)$ transcendental have infinite order. On the other hand, there exist equations of this form that possess one or more solutions of finite order. For instance: (a) $f(z)=$ $e^{-z}$ satisfies $f^{\prime \prime}+e^{z} f^{\prime}+\left(e^{2 z}-1\right) f=e^{z}-1$, and (b) $f(z)=c_{1} \sin z+c_{2} \cos z+e^{z}$ (where $c_{1}$ and $c_{2}$ are arbitrary constants) satisfies $f^{\prime \prime \prime}+e^{z} f^{\prime \prime}+f^{\prime}+e^{z} f=2 e^{z}+2 e^{2 z}$.

Two natural questions are:

(i) What conditions on $A_{0}(z), A_{1}(z), \ldots, A_{n-1}(z)$, and $H(z)$ will guarantee that every solution of (1.1) has infinite order?

(ii) If (1.1) possesses a solution $f$ of finite order, then how do the properties of $A_{0}(z), A_{1}(z), \ldots, A_{n-1}(z)$, and $H(z)$ affect the properties of $f$ ?

1991 Mathematics Subject Classification: Primary 34A20.

doi:10.5186/aasfm.1992.1722 
In this paper we obtain results on these two questions. We mention that [9] contains results on these two questions for equation (1.2) when $n=2$. See also [11].

We note that if $A_{0}(z), A_{1}(z), \ldots, A_{n-1}(z)$ are polynomials and $H(z)$ is of finite order, then [7, Lemma 2] every solution of (1.1) will have finite order. This result follows by using the method of variation of parameters together with the well-known result ([16, p. 108], [17, p. 65-68]) that if all the coefficients of (1.2) are polynomials, then every solution of (1.2) has finite order.

Some recent papers that investigate the properties of solutions of (1.1) in the case when $A_{0}(z), A_{1}(z), \ldots, A_{n-1}(z)$ are polynomials include [1], [5], [7], and [12].

\section{Statement of the main results}

For $w(z)$ an entire function, we let $\varrho(w)$ denote the order of $w$.

Our first result shows that if $f$ is a transcendental finite order solution of (1.1), and if the growth of one particular coefficient $A_{q}(z)$ dominates the growth of all the other coefficients in an angle, then $f^{(q)}$ will satisfy a certain growth condition in the angle.

Theorem 1. Let $\mu, \theta_{1}$, and $\theta_{2}$ be real constants satisfying $\mu>0$ and $\theta_{1}<\theta_{2}$. Suppose in (1.1) that there exists a unique coefficient $A_{q}(z)$ such that for any $\theta \in\left(\theta_{1}, \theta_{2}\right)$ there exist real constants $\alpha=\alpha(\theta)$ and $\beta=\beta(\theta)$ satisfying $0 \leq \beta<\alpha$, so that the following conditions hold as $z \rightarrow \infty$ along $\arg z=\theta$ :

$$
\begin{aligned}
& \left|A_{q}(z)\right| \geq \exp \left\{(\alpha+o(1))|z|^{\mu}\right\} \\
& \left|A_{k}(z)\right| \leq \exp \left\{(\beta+o(1))|z|^{\mu}\right\}
\end{aligned}
$$

for all $k \neq q$, and

$$
|H(z)| \leq \exp \left\{(\beta+o(1))|z|^{\mu}\right\} .
$$

Assume that $\alpha(\theta)$ and $\beta(\theta)$ are continuous functions on $\theta_{1}<\theta<\theta_{2}$.

Suppose that $f$ is a transcendental solution of (1.1) with $\varrho(f)<\infty$. If $l \geq q$ is an integer, then for any $\theta \in\left(\theta_{1}, \theta_{2}\right)$ we have

$$
\left|f^{(l)}(z)\right| \leq \exp \left\{-(\alpha-\beta+o(1))|z|^{\mu}\right\}
$$

as $z \rightarrow \infty$ along $\arg z=\theta$, where $\alpha=\alpha(\theta)$ and $\beta=\beta(\theta)$. 
It is easy to construct examples which show that the inequality (2.4) is sharp in the sense that we cannot replace the constant " $\alpha-\beta$ " by a larger constant. For example: (i) $f(z)=z^{2}+e^{-3 z}$ satisfies the equation

$$
f^{(v)}+\left(e^{z}+3\right) f^{(i v)}-e^{4 z} f^{\prime \prime \prime}-9 e^{z} f^{\prime \prime}+(2-3 z) f^{\prime}+6 f=4 z+9 e^{z}+9 z e^{-3 z}
$$

and (ii) $f(z)=1+e^{(b-a) z}$ satisfies the equation

$$
f^{(i v)}+e^{b z} f^{\prime \prime \prime}-(b-a)^{2} f^{\prime \prime}+(b-a) e^{a z} f^{\prime}-(b-a)^{3} e^{b z} f=(1+a-b)(b-a)^{2} e^{b z}
$$

where $a$ and $b$ are constants that satisfy $0 \leq b<a$.

We cannot switch $A_{q}(z)$ and $H(z)$ in the conditions (2.1) and (2.3) since, for example, $f(z)=\sin z$ satisfies $f^{\prime \prime}+f^{\prime}+(1+\sin z) f=\sin ^{2} z+\cos z$.

If in Theorem $1, f$ is a polynomial solution of (1.1), then $f^{(q)} \equiv 0$ would follow. We eliminate this trivial case by assuming that $f$ is transcendental in Theorem 1 (and in Theorem 2 below).

By combining Theorem 1, the Phragmén-Lindelöf theorem [14, pp. 270-271], and Liouville's theorem, we obtain the following two corollaries.

Corollary 1. Let $\theta_{1}, \theta_{2}, \ldots, \theta_{m}$ be a finite set of real numbers that satisfy $\theta_{1}<\theta_{2}<\cdots<\theta_{m}=\theta_{1}+2 \pi$. Suppose that for each $i=1,2, \ldots, m-1$, there exists in (1.1) one particular coefficient $A_{q_{i}}(z)$ and a corresponding constant $\mu_{i}>0$, such that for any $\theta \in\left(\theta_{i}, \theta_{i+1}\right)$ there exist constants $\alpha_{i}=\alpha_{i}(\theta)$ and $\beta_{i}=\beta_{i}(\theta)$ satisfying $0 \leq \beta_{i}<\alpha_{i}$, so that the following conditions hold as $z \rightarrow \infty$ along $\arg z=\theta$ :

$$
\begin{aligned}
&\left|A_{q_{i}}(z)\right| \geq \exp \left\{\left(\alpha_{i}+o(1)\right)|z|^{\mu_{i}}\right\} \\
&\left|A_{k}(z)\right| \leq \exp \left\{\left(\beta_{i}+o(1)\right)|z|^{\mu_{i}}\right\}
\end{aligned}
$$

for all $k \neq q_{i}$, and

$$
|H(z)| \leq \exp \left\{\left(\beta_{i}+o(1)\right)|z|^{\mu_{i}}\right\} .
$$

For each $i=1,2, \ldots, m-1$, assume that $\alpha_{i}(\theta)$ and $\beta_{i}(\theta)$ are continuous functions on $\theta_{i}<\theta<\theta_{i+1}$.

Then every transcendental solution $f$ of (1.1) satisfies $\varrho(f)=\infty$.

Corollary 2. Let $\theta_{1}, \theta_{2}, \ldots, \theta_{m}$ be a finite set of real numbers that satisfy $\theta_{1}<\theta_{2}<\cdots<\theta_{m}=\theta_{1}+2 \pi$. Suppose in (1.1) that there exists a unique coefficient $A_{q}(z)$ and a corresponding constant $\mu>0$, such that for any $\varepsilon>0$ there exists a constant $\alpha=\alpha(\varepsilon)>0$ so that

$$
\left|A_{q}(z)\right| \geq \exp \left\{(\alpha+o(1))|z|^{\mu}\right\}
$$

as $z \rightarrow \infty$ in $\theta_{i}+\varepsilon<\arg z<\theta_{i+1}-\varepsilon$ for $i=1,2, \cdots, m-1$.

If in (1.1), $\varrho\left(A_{k}\right)<\mu$ for all $k \neq q$, and $\varrho(H)<\mu$, then every transcendental solution $f$ of $(1.1)$ satisfies $\varrho(f)=\infty$. 
By Corollary 1, all solutions of the following equations have infinite order:

$$
\begin{gathered}
f^{\prime \prime}+(\sin z) f^{\prime}+(\sin z)^{2} f=\cos z \\
f^{(i v)}+e^{2 i z} f^{\prime \prime \prime}+e^{z} f^{\prime \prime}+e^{-(1+i) z} f^{\prime}+f=z^{2}+1, \\
f^{\prime \prime \prime}+e^{z^{3}} f^{\prime \prime}+(\sin z) f^{\prime}+e^{z^{2}} f=\cos \sqrt{z} .
\end{gathered}
$$

If $l \geq 4$ is an integer, then by Corollary 2 (or Corollary 1 ), all solutions of

$$
f^{(i v)}+e^{z^{2}} f^{\prime \prime \prime}+\left(\cos z^{l}\right) f^{\prime \prime}+e^{-z^{3}} f^{\prime}+z f=e^{z}
$$

have infinite order. Furthermore, given any real number $\mu>1$, the Mittag-Leffler function $\left[3\right.$, p. 50] can be used to construct an entire function $A_{q}(z)$ in Corollary 2 with $\varrho\left(A_{q}\right)=\mu$.

Corollary 1 shows that if for each $i$ the growth of one particular coefficient $A_{q_{i}}(z)$ in (1.1) dominates the growth of the other coefficients along any ray $\arg z=$ $\theta$ satisfying $\theta_{i}<\theta<\theta_{i+1}$, then all transcendental solutions of (1.1) have infinite order. Theorem 2 below shows that we can weaken this hypothesis and still get the same conclusion. More specifically, Theorem 2 shows that if we have such a dominating coefficient $A_{q_{i}}(z)$ in at least one of the sectors $\theta_{i}<\arg z<\theta_{i+1}$, and if we have certain other growth conditions in any sector $\theta_{i}<\arg z<\theta_{i+1}$ where such a dominating coefficient does not exist, then all transcendental solutions of (1.1) will have infinite order.

Theorem 2. Let $\theta_{1}, \theta_{2}, \ldots, \theta_{m}$ be a finite set of real numbers that satisfy $\theta_{1}<\theta_{2}<\cdots<\theta_{m}=\theta_{1}+2 \pi$. For equation (1.1), suppose that for each $i=1,2, \ldots, m-1$, either condition (a) or condition (b) below holds for the interval $\left(\theta_{i}, \theta_{i+1}\right)$, and suppose also that condition (a) holds for at least one interval:

(a) There exists one particular coefficient $A_{q_{i}}(z)$ and a corresponding constant $\mu_{i}>0$, such that for any $\theta \in\left(\theta_{i}, \theta_{i+1}\right)$ there exist constants $\alpha_{i}=\alpha_{i}(\theta)$ and $\beta_{i}=\beta_{i}(\theta)$ satisfying $0 \leq \beta_{i}<\alpha_{i}$, so that (2.7), (2.8), and (2.9) hold as $z \rightarrow \infty$ along $\arg z=\theta$. Assume that $\alpha_{i}(\theta)$ and $\beta_{i}(\theta)$ are continuous functions on $\theta_{i}<$ $\theta<\theta_{i+1}$.

(b) Condition (b) consists of when (2.14) and (2.16) below both hold for the interval $\left(\theta_{i}, \theta_{i+1}\right)$. For any $\nu$ satisfying $0 \leq \nu \leq n-1$ and for any $\theta \in\left(\theta_{i}, \theta_{i+1}\right)$, we have

$$
z^{n-\nu} A_{\nu}(z) \rightarrow 0
$$


as $z \rightarrow \infty$ along $\arg z=\theta$. To describe (2.16) we first set $\mu=\max \mu_{j}$ where this maximum is taken over those values of $j$ such that condition (a) holds for the interval $\left(\theta_{j}, \theta_{j+1}\right)$. Then set

$$
\tau=\sup _{\theta, k}\left\{\alpha_{k}(\theta)-\beta_{k}(\theta)\right\}
$$

where this supremum is taken over all $\theta \in\left(\theta_{k}, \theta_{k+1}\right)$ and all $k$ such that condition (a) holds for the interval $\left(\theta_{k}, \theta_{k+1}\right)$ with $\mu_{k}=\mu$. Then the second part of condition (b) is that there exists a constant $\lambda$ that satisfies $0 \leq \lambda<\tau$, such that for any $\theta \in\left(\theta_{i}, \theta_{i+1}\right)$ we have

$$
|H(z)| \leq \exp \left\{(\lambda+o(1))|z|^{\mu}\right\}
$$

as $z \rightarrow \infty$ along $\arg z=\theta$.

Then every transcendental solution $f$ of (1.1) satisfies $\varrho(f)=\infty$.

By Theorem 2, all solutions of the equation

$$
f^{\prime \prime \prime}+e^{z} f^{\prime \prime}+e^{3 z} f^{\prime}+e^{2 z} f=e^{z}+1
$$

have infinite order. In this example condition (a) holds for the interval $(-\pi / 2, \pi / 2)$, while condition (b) holds for the interval $(\pi / 2,3 \pi / 2)$ with $\mu=1, \tau=1$, and $\lambda=0$.

We can generalize this example. The next result is an immediate corollary of Theorem 2.

Corollary 3. Let $P_{0}(z), P_{1}(z), \ldots, P_{n-1}(z)$, and $P(z)$ be polynomials in $z$ with $n \geq 1$ and $P(z) \not \equiv 0$, and suppose that there exists one particular integer $q$ satisfying $0 \leq q \leq n-1$ such that $\operatorname{deg}\left(P_{q}\right)>\operatorname{deg}\left(P_{k}\right)$ for all $k \neq q$, $\operatorname{deg}\left(P_{q}\right)>\operatorname{deg}(P)$, and $P_{k}(0)=0$ for all $k=0,1, \ldots, n-1$. If $l \geq 1$ is a positive integer, then every transcendental solution $f$ of the equation

$$
f^{(n)}+P_{n-1}\left(e^{z^{l}}\right) f^{(n-1)}+\cdots+P_{1}\left(e^{z^{l}}\right) f^{\prime}+P_{0}\left(e^{z^{l}}\right) f=P\left(e^{z^{l}}\right)
$$

satisfies $\varrho(f)=\infty$.

Another illustration of Theorem 2 is that every solution of

$$
f^{\prime \prime}+\left(\frac{e^{z}-1}{z}\right)^{2} f^{\prime}+\left(\frac{e^{z}-1}{z}\right)^{3} f=e^{z}
$$

has infinite order. Of course, the earlier examples (2.10), (2.11), (2.12), and (2.13) for Corollary 1 are also examples of Theorem 2 since Corollary 1 is a corollary of both Theorem 2 and Theorem 1. 
Regarding condition (b) in Theorem 2, the examples in (2.5) and (2.6) illustrate that if condition (a) holds for some of the intervals $\left(\theta_{i}, \theta_{i+1}\right)$ but not all of the intervals, then in order to obtain the conclusion of Theorem 2 we must have some kind of condition(s) on the coefficients in (1.1) for the intervals $\left(\theta_{i}, \theta_{i+1}\right)$ where condition (a) does not hold.

Condition (a) in Theorem 2 is sharp in the sense that if condition (a) holds for every interval $\left(\theta_{i}, \theta_{i+1}\right), i=1,2, \ldots, m-1$, then we cannot replace the condition "0 $\leq \beta_{i}<\alpha_{i}$ " with " $0 \leq \beta_{i} \leq \alpha_{i}$ ". Consider, for example, that $f(z)=\sin z$ satisfies $f^{\prime \prime}-(\sin z) f^{\prime}+(\cos z) f=-\sin z$.

The inequality (2.16) in condition (b) of Theorem 2 is sharp in the sense that we cannot replace the condition " $0 \leq \lambda<\tau$ " with " $0 \leq \lambda \leq \tau$ " by the following example: For any real constant $c$ satisfying $0<c<1, f(z)=e^{-c z}$ satisfies the equation

$$
f^{\prime \prime}+\left(\frac{e^{z}-1}{z}\right)^{2} f^{\prime}+\left(\frac{e^{z}-1}{z}\right)^{3} f=c^{2} e^{-c z}-c e^{-c z}\left(\frac{e^{z}-1}{z}\right)^{2}+e^{-c z}\left(\frac{e^{z}-1}{z}\right)^{3} .
$$

In this example condition (a) holds for the interval $(-\pi / 2, \pi / 2),(2.14)$ holds for the interval $(\pi / 2,3 \pi / 2)$, but $(2.16)$ does not hold for the interval $(\pi / 2,3 \pi / 2)$ since $\lambda=\tau=c$ (with $\mu=1$ ).

We do not know whether the exponents " $n-\nu$ " in (2.14) are sharp or not. Regarding this question, we mention that $f(z)=e^{-z}$ satisfies the following two equations:

$$
f^{\prime \prime}+\left(e^{z}+1\right) f^{\prime}+e^{2 z} f=e^{z}-1
$$

$$
f^{\prime \prime}+\left(\frac{e^{z}-1}{z}\right)^{2} f^{\prime}+\left[\left(\frac{e^{z}-1}{z}\right)^{2}-1+e^{3 z}\right] f=e^{2 z} .
$$

We next consider the particular case where the orders of the coefficients in (1.1) are all less than $1 / 2$. We have the following result.

Theorem 3. Suppose in (1.1) that $\max \left\{\varrho\left(A_{1}\right), \varrho\left(A_{2}\right), \ldots, \varrho\left(A_{n-1}\right), \varrho(H)\right\}<$ $\varrho\left(A_{0}\right)<1 / 2$. Then every solution of (1.1) has infinite order.

In contrast to Theorem 3, there exist equations of the form (1.1) where

$$
\max _{0 \leq k \leq n-1} \varrho\left(A_{k}\right)<\varrho(H)<\frac{1}{2}
$$

which possess a solution of finite order (see Section 7). 


\section{Lemmas}

We shall use the lemmas in this section in the proofs of our results.

Lemma 1 [8]. Let $w(z)$ be a transcendental entire function of finite order $\varrho$, let $\Gamma=\left\{\left(k_{1}, j_{1}\right),\left(k_{2}, j_{2}\right), \ldots,\left(k_{m}, j_{m}\right)\right\}$ denote a finite set of distinct pairs of integers that satisfy $k_{i}>j_{i} \geq 0$ for $i=1,2, \ldots, m$, and let $\varepsilon>0$ be a given constant. Then the following two statements hold:

(i) There exists a set $E_{1} \subset[0,2 \pi)$ that has linear measure zero, such that if $\theta \in[0,2 \pi)-E_{1}$, then there is a constant $R=R(\theta)>0$ such that for all $z$ satisfying $\arg z=\theta$ and $|z| \geq R$, and for all $(k, j) \in \Gamma$, we have

$$
\left|\frac{w^{(k)}(z)}{w^{(j)}(z)}\right| \leq|z|^{(k-j)(\varrho-1+\varepsilon)}
$$

(ii) There exists a set $E_{2} \subset[0, \infty)$ that has finite linear measure such that for all $z$ satisfying $|z| \notin E_{2}$ and for all $(k, j) \in \Gamma$, we have

$$
\left|\frac{w^{(k)}(z)}{w^{(j)}(z)}\right| \leq|z|^{(k-j)(\varrho+\varepsilon)}
$$

The next result can be deduced by using standard reasoning with the classical Phragmén-Lindelöf theorem (see, e.g., [13, p. 49]).

Lemma 2. Suppose that $w(z)$ is an entire function where $\varrho(w)<\infty$. Let $\mu, \theta_{1}$, and $\theta_{2}$ be real constants satisfying $\mu>0$ and $\theta_{1}<\theta_{2}$, and let $\lambda(\theta)$ be a continuous function on $\theta_{1}<\theta<\theta_{2}$ where $\lambda(\theta)>0$ for all $\theta$. Suppose that there exists a set $E \subset \mathbf{R}$ that has linear measure zero such that for any $\theta \in\left(\theta_{1}, \theta_{2}\right)-E$ we have

$$
|w(z)| \leq \exp \left\{-(\lambda+o(1))|z|^{\mu}\right\}
$$

as $z \rightarrow \infty$ along $\arg z=\theta$, where $\lambda=\lambda(\theta)$.

Then for every $\theta \in\left(\theta_{1}, \theta_{2}\right)$, (3.1) holds as $z \rightarrow \infty$ along $\arg z=\theta$, where $\lambda=\lambda(\theta)$.

The next lemma is a generalization of Lemma 1 in [10].

Lemma 3. Let $\theta$ and $R_{0} \geq 0$ be real constants. Let $w(z)$ be analytic on the ray $\left\{z: \arg z=\theta\right.$ and $\left.|z| \geq R_{0}\right\}$, and let $\eta(r)$ be a non-decreasing positive function on the interval $R_{0} \leq r<\infty$. Let $l \geq 1$ be an integer and set

$$
G(r)=\frac{\left|w^{(l)}\left(r e^{i \theta}\right)\right|}{\eta(r)} .
$$


If $G(r)$ is unbounded as $r \rightarrow+\infty$, then there exists an infinite sequence $\left\{r_{j}\right\}$ satisfying $R_{0}<r_{j}<\infty$ and $r_{j} \rightarrow+\infty$, such that as $r_{j} \rightarrow+\infty$ we have both

$$
G\left(r_{j}\right) \rightarrow+\infty
$$

and

$$
\left|\frac{w^{(l)}\left(r_{j} e^{i \theta}\right)}{w^{(k)}\left(r_{j} e^{i \theta}\right)}\right| \geq(1+o(1)) \frac{1}{\left(r_{j}\right)^{l-k}}
$$

for each $k=0,1, \ldots, l-1$.

The exponent $l-k$ on the right side of (3.4) is sharp by consideration of the functions $f_{1}(z)=Q(z)+e^{z}$ (when $\left.\pi / 2<\theta<3 \pi / 2\right)$ and $f_{2}(z)=Q(z)$ where $Q(z)$ is a non-constant polynomial, with $\eta(r) \equiv 1$ in (3.2).

Proof of Lemma 3. Since $G(r)$ is unbounded as $r \rightarrow \infty$, it follows that there exists an infinite sequence $\left\{r_{j}\right\}$ where $R_{0}<r_{j}<\infty$ and $r_{j} \rightarrow \infty$, such that $G\left(r_{j}\right) \rightarrow \infty$ as $r_{j} \rightarrow \infty$ and

$$
G(r) \leq G\left(r_{j}\right)
$$

for all $r$ satisfying $R_{0} \leq r \leq r_{j}$. Thus (3.3) holds, and so it remains to show that (3.4) holds.

Set $z_{j}=r_{j} e^{i \theta}$, set $\zeta_{0}=R_{0} e^{i \theta}$, and let $\left[\zeta_{0}, z_{j}\right]$ denote the line segment joining $\zeta_{0}$ and $z_{j}$. We claim that for $k=0,1, \ldots, l-1$, we have

$$
\begin{aligned}
\left|w^{(k)}(z)\right| \leq & \left|w^{(k)}\left(\zeta_{0}\right)\right|+|z|\left|w^{(k+1)}\left(\zeta_{0}\right)\right| \\
& +\cdots+|z|^{l-k-1}\left|w^{(l-1)}\left(\zeta_{0}\right)\right|+|z|^{l-k}\left|w^{(l)}\left(z_{j}\right)\right|
\end{aligned}
$$

for all $z \in\left[\zeta_{0}, z_{j}\right]$. We will then obtain (3.4) by setting $z=z_{j}$ in (3.6) and noting that $w^{(l)}\left(z_{j}\right) \rightarrow \infty$ as $j \rightarrow \infty$, which follows from (3.3) and (3.2). We now show that (3.6) holds for each $k=0,1, \ldots, l-1$.

Using (3.5) and (3.2), we first note that for $z \in\left[\zeta_{0}, z_{j}\right]$ :

$$
\begin{aligned}
\left|w^{(l-1)}(z)\right| & =\left|w^{(l-1)}\left(\zeta_{0}\right)+\int_{\zeta_{0}}^{z} w^{(l)}(\zeta) d \zeta\right| \leq\left|w^{(l-1)}\left(\zeta_{0}\right)\right|+\int_{R_{0}}^{|z|} G(r) \eta(r) d r \\
& \leq\left|w^{(l-1)}\left(\zeta_{0}\right)\right|+\left(|z|-R_{0}\right) G\left(r_{j}\right) \eta\left(r_{j}\right) \leq\left|w^{(l-1)}\left(\zeta_{0}\right)\right|+|z|\left|w^{(l)}\left(z_{j}\right)\right| .
\end{aligned}
$$

Thus (3.6) holds for $k=l-1$.

For the induction step, we next suppose that $m$ is any fixed integer satisfying $1 \leq m \leq l-1$ and that (3.6) holds for $k=m$. We will show that (3.6) holds for $k=m-1$. Since (3.6) holds for $k=m$, we have for $z \in\left[\zeta_{0}, z_{j}\right]$ :

$$
\begin{aligned}
\left|w^{(m)}(z)\right| \leq & \left|w^{(m)}\left(\zeta_{0}\right)\right|+|z|\left|w^{(m+1)}\left(\zeta_{0}\right)\right| \\
& +\cdots+|z|^{l-m-1}\left|w^{(l-1)}\left(\zeta_{0}\right)\right|+|z|^{l-m}\left|w^{(l)}\left(z_{j}\right)\right| .
\end{aligned}
$$


Using (3.7) and (3.5), we obtain for $z \in\left[\zeta_{0}, z_{j}\right]$ :

$$
\begin{aligned}
\left|w^{(m-1)}(z)\right|= & \left|w^{(m-1)}\left(\zeta_{0}\right)+\int_{\zeta_{0}}^{z} w^{(m)}(\zeta) d \zeta\right| \\
\leq & \left|w^{(m-1)}\left(\zeta_{0}\right)\right|+\left(|z|-R_{0}\right)\left\{\left|w^{(m)}\left(\zeta_{0}\right)\right|+|z|\left|w^{(m+1)}\left(\zeta_{0}\right)\right|\right. \\
& \left.\quad+\cdots+|z|^{l-m-1}\left|w^{(l-1)}\left(\zeta_{0}\right)\right|+|z|^{l-m}\left|w^{(l)}\left(z_{j}\right)\right|\right\} .
\end{aligned}
$$

This yields (3.6) for $k=m-1$.

Hence we have now proven by induction that (3.6) holds for each $k=$ $0,1, \ldots, l-1$, and the proof of Lemma 3 is now complete.

Lemma 4 below is used in the proof of Theorem 3, while Lemma 5 below is used in the proof of the example in Section 7.

Lemma 4 [2]. Let $w$ be an entire function of order $\varrho$ where $0<\varrho<1 / 2$, and let $\varepsilon>0$ be a given constant. Then there exists a set $S \subset[0, \infty)$ that has upper density at least $1-2 \varrho$ such that $|w(z)|>\exp \left(|z|^{\varrho-\varepsilon}\right)$ for all $z$ satisfying $|z| \in S$.

For a definition of "upper density", see, for example, [2, p. 679].

Lemma 5 [4]. If $w$ is an entire function with lower order $\lambda>0$, then there exists a curve $\Gamma$ that goes from a finite point to $\infty$ for which

$$
\liminf _{z \rightarrow \infty, z \in \Gamma} \frac{\ln \ln |w(z)|}{\ln |z|} \geq \min \left(\frac{1}{2}, \lambda\right) \text {. }
$$

\section{Proof of Theorem 1}

From (1.1),

$$
\begin{aligned}
f^{(q)}\left[\frac{f^{(n)}}{f^{(q)}} \frac{1}{A_{q}}\right. & +\frac{f^{(n-1)}}{f^{(q)}} \frac{A_{n-1}}{A_{q}}+\cdots+\frac{f^{(q+1)}}{f^{(q)}} \frac{A_{q+1}}{A_{q}} \\
& \left.+1+\frac{f^{(q-1)}}{f^{(q)}} \frac{A_{q-1}}{A_{q}}+\cdots+\frac{f}{f^{(q)}} \frac{A_{0}}{A_{q}}\right]=\frac{H}{A_{q}} .
\end{aligned}
$$

From Lemma 1(i) it follows that there exist a constant $c>0$ and a set $E \subset \mathbf{R}$ with linear measure zero, such that if $\theta \in \mathbf{R}-E$ then for any $k$ satisfying $q<k \leq n-1$ we have

$$
\left|\frac{f^{(k)}(z)}{f^{(q)}(z)}\right|=o(1)|z|^{c}
$$

as $z \rightarrow \infty$ along $\arg z=\theta$. 
We now let $\psi \in\left(\theta_{1}, \theta_{2}\right)-E$, and we assume that $f^{(q)}(z)$ is unbounded on $\arg z=\psi$. Then from Lemma 3, there exists an infinite sequence of points $z_{j}=r_{j} e^{i \psi}$ where $r_{j} \rightarrow \infty$, such that

$$
f^{(q)}\left(z_{j}\right) \rightarrow \infty
$$

and

$$
\left|\frac{f^{(k)}\left(z_{j}\right)}{f^{(q)}\left(z_{j}\right)}\right| \leq(1+o(1))\left|z_{j}\right|^{q-k}
$$

as $z_{j} \rightarrow \infty$, for all $k$ satisfying $0 \leq k \leq q-1$.

Combining (4.2), (4.4), (2.1), (2.2), and (2.3) together with (4.1) yields $f^{(q)}\left(z_{j}\right) \rightarrow 0$ as $z_{j} \rightarrow \infty$. This contradicts (4.3). Hence $f^{(q)}(z)$ must be bounded on $\arg z=\psi$.

It easily follows that for all $k$ satisfying $0 \leq k \leq q-1$,

$$
\left|f^{(k)}(z)\right|=O\left(|z|^{q-k}\right)
$$

as $z \rightarrow \infty$ along $\arg z=\psi$.

Now from (1.1) we have

$$
\begin{gathered}
f^{(q)}\left[\frac{f^{(n)}}{f^{(q)}} \frac{1}{A_{q}}+\frac{f^{(n-1)}}{f^{(q)}} \frac{A_{n-1}}{A_{q}}+\cdots+\frac{f^{(q+1)}}{f^{(q)}} \frac{A_{q+1}}{A_{q}}+1\right] \\
=\frac{H}{A_{q}}-\left[f^{(q-1)} \frac{A_{q-1}}{A_{q}}+\cdots+f \frac{A_{0}}{A_{q}}\right] .
\end{gathered}
$$

Combining (2.1), (2.2), (2.3), (4.2), (4.5), and (4.6) we find that

$$
\left|f^{(q)}(z)\right| \leq \exp \left\{-(\alpha-\beta+o(1))|z|^{\mu}\right\}
$$

as $z \rightarrow \infty$ along $\arg z=\psi$, where $\alpha=\alpha(\psi)$ and $\beta=\beta(\psi)$.

Since $\psi \in\left(\theta_{1}, \theta_{2}\right)-E$ was arbitrarily chosen, we have thus shown that for any $\theta \in\left(\theta_{1}, \theta_{2}\right)-E,(4.7)$ holds as $z \rightarrow \infty$ along $\arg z=\theta$, with $\alpha=\alpha(\theta)$ and $\beta=\beta(\theta)$. By combining this with $\varrho\left(f^{(q)}\right)<\infty$ and Lemma 2, we obtain that for any $\theta \in\left(\theta_{1}, \theta_{2}\right),(4.7)$ holds as $z \rightarrow \infty$ along $\arg z=\theta$, with $\alpha=\alpha(\theta)$ and $\beta=\beta(\theta)$. This proves (2.4) for $l=q$. We can then use the fact that (2.4) holds for $l=q$ together with the Phragmén-Lindelöf theorem [14, p. 214] and the Cauchy integral formula to deduce that (2.4) holds for all $l>q$. This proves Theorem 1. 


\section{Proof of Theorem 2}

Let $f$ be a transcendental solution of equation (1.1). We make the assumption that $\varrho(f)<\infty$.

Now suppose that $p$ is a value such that condition (a) holds for $\left(\theta_{p}, \theta_{p+1}\right)$ with $\mu_{p}=\mu$ and such that the constant $\tau$ in (2.15) satisfies

$$
\tau=\sup _{\theta \in\left(\theta_{p}, \theta_{p+1}\right)}\left\{\alpha_{p}(\theta)-\beta_{p}(\theta)\right\} .
$$

Then from Theorem 1, we obtain that for any $\theta \in\left(\theta_{p}, \theta_{p+1}\right)$,

$$
\left|f^{(n)}(z)\right| \leq \exp \left\{-\left(\alpha_{p}-\beta_{p}+o(1)\right)|z|^{\mu}\right\}
$$

as $z \rightarrow \infty$ along $\arg z=\theta$, where $\alpha_{p}=\alpha_{p}(\theta)$ and $\beta_{p}=\beta_{p}(\theta)$. From (5.2) and $[15$, p. 273] we obtain

$$
0<\mu \leq \varrho(f)<\infty
$$

Now let $\xi$ be a constant that satisfies

$$
\lambda<\xi<\tau .
$$

For fixed real values of $\theta$ we will consider the quantity

$$
\frac{\left|f^{(n)}\left(r e^{i \theta}\right)\right|}{\exp \left(\xi r^{\mu}\right)}
$$

as $r \rightarrow \infty$. Below we shall use the Phragmén-Lindelöf theory (i.e., the classical Phragmén-Lindelöf theorem and related results); see, for example, [3], [13], and [14].

We will now show that there must exist a real number $\psi$ satisfying $\psi \neq \theta_{i}$ for $i=1,2, \ldots, m$, and $\theta_{1}<\psi<\theta_{m}=\theta_{1}+2 \pi$, such that for $\psi=\theta$ the quantity (5.5) is unbounded as $r \rightarrow \infty$. We will prove this by contradiction. Suppose that this were not true. Since $\varrho(f)=\varrho\left(f^{(n)}\right)<\infty$, we can then deduce from the Phragmén-Lindelöf theory that $\varrho\left(f^{(n)}\right) \leq \mu$. Then from (5.3), $\varrho\left(f^{(n)}\right)=\mu$. Furthermore, it follows from the above assumption and the Phragmén-Lindelö theory that the type $\sigma$ of $f^{(n)}$ satisfies

$$
\sigma \leq \xi<\infty
$$

Now let

$$
h(\theta)=\limsup _{r \rightarrow \infty} \frac{\ln \left|f^{(n)}\left(r e^{i \theta}\right)\right|}{r^{\mu}}
$$


denote the Phragmén-Lindelöf indicator function of $f^{(n)}$ (see, e.g., [3], [13], and [14]). Since $\sigma<\infty, h(\theta)$ is a continuous function for all $\theta$ (see, e.g., [14, p. 272 and p. 275]). Thus from (5.1), (5.2), (5.4), and (5.7) it follows that there must exist a real constant $\phi \in\left(\theta_{p}, \theta_{p+1}\right)$ such that

$$
h(\phi)<-\xi<0 .
$$

Hence from (5.8) and the theory of the indicator function $h(\theta)$ [14, p. 276-277], we deduce that $\sigma \geq|h(\phi)|>\xi$. This contradicts (5.6). Hence the above assumption must be false, and so there must exist a real number $\psi$ satisfying $\psi \neq \theta_{i}$ for $i=1,2, \ldots, m$, and $\theta_{1}<\psi<\theta_{m}=\theta_{1}+2 \pi$, such that

$$
\limsup _{r \rightarrow \infty} \frac{\left|f^{(n)}\left(r e^{i \psi}\right)\right|}{\exp \left(\xi r^{\mu}\right)}=\infty
$$

Now if $j$ is any value where condition (a) holds for $\left(\theta_{j}, \theta_{j+1}\right)$, then from Theorem 1 we obtain that for any $\theta \in\left(\theta_{j}, \theta_{j+1}\right), f^{(n)}(z) \rightarrow 0$ as $z \rightarrow \infty$ along $\arg z=\theta$. Hence from (5.9) it follows that (2.14) and (2.16) must both hold as $z \rightarrow \infty$ along $\arg z=\psi$.

Now from (1.1),

$$
1+A_{n-1}(z) \frac{f^{(n-1)}}{f^{(n)}}+\cdots+A_{0}(z) \frac{f}{f^{(n)}}=\frac{H(z)}{f^{(n)}} .
$$

Because of (5.9), we can apply Lemma 3 to $f(z)$ with $\psi=\theta$ and $\eta(r)=$ $\exp \left(\xi r^{\mu}\right)$. Then from (5.9) and Lemma 3 it follows that there exists a sequence $r_{j} \rightarrow \infty$ such that

$$
\frac{\left|f^{(n)}\left(r_{j} e^{i \psi}\right)\right|}{\exp \left(\xi r_{j}^{\mu}\right)} \rightarrow \infty
$$

as $r_{j} \rightarrow \infty$, and

$$
\left|\frac{f^{(n)}\left(r_{j} e^{i \psi}\right)}{f^{(\nu)}\left(r_{j} e^{i \psi}\right)}\right| \geq \frac{1+o(1)}{\left(r_{j}\right)^{n-\nu}}
$$

as $r_{j} \rightarrow \infty$, for $\nu=0,1, \ldots, n-1$.

Now by combining (5.4), (5.11), (5.12), (2.14), and (2.16), it can be deduced that (5.10) will yield the contradiction $1=0$ as $r_{j} \rightarrow \infty$. This contradiction implies that our original assumption that $\varrho(f)<\infty$ must be false. This proves Theorem 2. 


\section{Proof of Theorem 3}

Suppose that $f$ is a solution of $(1.1)$ with $\varrho(f)<\infty$. Then from Lemma 1(ii), there exist a constant $c>0$ and a set $E \subset[0, \infty)$ of finite linear measure, such that

$$
\left|\frac{f^{(k)}(z)}{f(z)}\right| \leq|z|^{c}
$$

for all $z$ satisfying $|z| \notin E$ and for all $k=1,2, \ldots, n$. Let $\alpha$ and $\beta$ be any fixed positive constants that satisfy

$$
\max \left\{\varrho\left(A_{1}\right), \varrho\left(A_{2}\right), \ldots, \varrho\left(A_{n-1}\right), \varrho(H)\right\}<\beta<\alpha<\varrho\left(A_{0}\right) .
$$

From Lemma 4 there exists a set $S \subset[0, \infty)$ that has upper density at least $1-2 \varrho\left(A_{0}\right)>0$ such that

$$
\left|A_{0}(z)\right|>\exp \left\{|z|^{\alpha}\right\}
$$

for all $z$ satisfying $|z| \in S$. From (6.2) we obtain that there exists a constant $R>0$ such that the following conditions hold for $|z|>R$ :

$$
\left|A_{k}(z)\right| \leq \exp \left\{|z|^{\beta}\right\}
$$

for $k=1,2, \ldots, n-1$, and

$$
|H(z)| \leq \exp \left\{|z|^{\beta}\right\} .
$$

Since $E$ has finite linear measure and $S$ has positive upper density, there exists an infinite sequence $r_{j} \rightarrow \infty$ such that

$$
r_{j} \in\{r: r>R\} \cap S \quad \text { and } \quad r_{j} \notin E
$$

for all $j$.

From (1.1) we have

$$
f\left[\frac{f^{(n)}}{f} \frac{1}{A_{0}(z)}+\frac{f^{(n-1)}}{f} \frac{A_{n-1}(z)}{A_{0}(z)}+\cdots+\frac{f^{\prime}}{f} \frac{A_{1}(z)}{A_{0}(z)}+1\right]=\frac{H(z)}{A_{0}(z)} .
$$

Then from (6.1), (6.2), (6.3), (6.4), (6.5), (6.6), and (6.7), we deduce that for all $z$ satisfying $|z|=r_{j}$ we have

$$
f(z) \rightarrow 0 \quad \text { as } \quad r_{j} \rightarrow \infty .
$$

Relation (6.8) and the maximum modulus principle imply that $f \equiv 0$. But $f \equiv 0$ contradicts that $f$ satisfies (1.1). This contradiction proves Theorem 3 . 


\section{An example}

In contrast to Theorem 3 , we will now show that there exist entire functions $A_{0}, A_{1}, H$, and $f$ with $\max \left\{\varrho\left(A_{0}\right), \varrho\left(A_{1}\right)\right\}<\varrho(H)<1 / 2$ and $\varrho(f)<\infty$ such that $f^{\prime \prime}+A_{1}(z) f^{\prime}+A_{0}(z) f=H(z)$.

Let $A_{0}(z)$ be any entire function such that $0<\varrho\left(A_{0}\right)<1 / 2$ and where the lower order of $A_{0}$ equals $\varrho\left(A_{0}\right)$. Then let $A_{1}$ and $f$ be any transcendental entire functions that satisfy

$$
\varrho\left(A_{1}\right)<\varrho\left(A_{0}\right)<\varrho(f)<1 / 2 .
$$

Let $H(z)$ be defined by the equation

$$
f^{\prime \prime}+A_{1}(z) f^{\prime}+A_{0}(z) f=H(z) .
$$

From (7.2) and (7.1), $H(z)$ is entire and

$$
\varrho(H) \leq \varrho(f) .
$$

Now let $\alpha$ and $\beta$ be any fixed constants that satisfy

$$
\varrho\left(A_{1}\right)<\beta<\varrho\left(A_{0}\right)<\alpha<\varrho(f)<1 / 2 .
$$

From Lemma 4 there exists a set $S \subset[0, \infty)$ with positive upper density such that

$$
|f(z)|>e^{|z|^{\alpha}}
$$

for all $z$ satisfying $|z| \in S$. Since the lower order of $A_{0}$ equals $\varrho\left(A_{0}\right)$, we deduce from Lemma 5 and (7.4) that there exists a curve $\Gamma$ that goes from a finite point to $\infty$ such that

$$
\left|A_{0}(z)\right|>e^{|z|^{\beta}}
$$

for all $z \in \Gamma$.

We also know from Lemma 1(ii) that there exist a constant $c>0$ and a set $E \subset[0, \infty)$ of finite linear measure, such that

$$
\left|\frac{f^{\prime \prime}(z)}{f(z)}\right| \leq|z|^{c} \quad \text { and } \quad\left|\frac{f^{\prime}(z)}{f(z)}\right| \leq|z|^{c}
$$

for all $z$ satisfying $|z| \notin E$.

Now there exist arbitrarily large points $z_{0}$ that satisfy

$$
\left|z_{0}\right| \in S-E \quad \text { and } \quad z_{0} \in \Gamma \text {. }
$$

For such points $z_{0}$, we deduce from (7.2), (7.4), (7.5), (7.6), (7.7), and (7.8) that as $z_{0} \rightarrow \infty$ :

$$
\left|H\left(z_{0}\right)\right|=\left|f\left(z_{0}\right) A_{0}\left(z_{0}\right)\right|\left|\frac{f^{\prime \prime}\left(z_{0}\right)}{f\left(z_{0}\right) A_{0}\left(z_{0}\right)}+\frac{f^{\prime}\left(z_{0}\right) A_{1}\left(z_{0}\right)}{f\left(z_{0}\right) A_{0}\left(z_{0}\right)}+1\right|>\exp \left\{(1+o(1))\left|z_{0}\right|^{\alpha}\right\} .
$$

Thus $\varrho(H) \geq \alpha$. Since $\alpha$ can be arbitrarily close to $\varrho(f)$ in (7.4), $\varrho(H) \geq \varrho(f)$. Then from (7.3), $\varrho(H)=\varrho(f)$. Hence from (7.1), $\max \left\{\varrho\left(A_{0}\right), \varrho\left(A_{1}\right)\right\}<\varrho(H)<$ $1 / 2$, and the assertion is now proved. 


\section{References}

[1] BANK, S.: On the oscillation of solutions of non-homogeneous linear differential equations.

- Analysis 10, 1990, 265-293.

[2] Besicovitch, A.S.: On integral functions of order < 1. - Math. Ann. 97, 1927, 677-695.

[3] Cartwright, M.L.: Integral functions. - Cambridge University Press, London, 1962.

[4] Chang, K.-H.: Asymptotic values of entire and meromorphic functions. - Scientia Sinica $20,1977,720-739$.

[5] Frank, G., and S. Hellerstein: On the meromorphic solutions of non-homogeneous linear differential equations with polynomial coefficients. - Proc. London Math. Soc. (3) $53,1986,407-428$.

[6] Frei, M.: Sur l'ordre des solutions entières d'une équation différentielle linéaire. - C. R. Acad. Sci. Paris 236, 1953, 38-40.

[7] Gao SHI'AN: On the complex oscillation of solutions of non-homogeneous linear differential equations with polynomial coefficients. - Comment. Math. Univ. St. Paul. 38, 1989, $11-20$.

[8] Gundersen, G.: Estimates for the logarithmic derivative of a meromorphic function, plus similar estimates. - J. London Math. Soc. (2) 37, 1988, 88-104.

[9] Gundersen, G.: Finite order solutions of second order linear differential equations. Trans. Amer. Math. Soc. 305, 1988, 415-429.

[10] Gundersen, G.: The deficiencies of meromorphic solutions of certain algebraic differential equations. - Results in Math. 16, 1989, 54-76.

[11] Hellerstein, S., J. Miles, and J. Rossi: On the growth of solutions of $f^{\prime \prime}+g f^{\prime}+h f=0$. - Trans. Amer. Math. Soc. 324, 1991, 693-706.

[12] LAine, I.: A note on the complex oscillation theory of non-homogeneous linear differential equations. - Results in Math. 18, 1990, 282-285.

[13] Levin, B. JA.: Distribution of zeros of entire functions. - Revised edition, Translations of Mathematical Monographs, Volume 5, American Mathematical Society, Providence, Rhode Island, 1980.

[14] Markushevich, A.: Theory of functions of a complex variable, Volume II. - Translated by R. Silverman, Prentice-Hall, Englewood Cliffs, N.J., 1965.

[15] Titchmarsh, E.C.: The theory of functions. - Second edition, Oxford University Press, London, 1968.

[16] Valiron, G.: Lectures on the general theory of integral functions. - Translated by E.F. Collingwood, Chelsea, New York, 1949.

[17] Wiтtich, H.: Neuere Untersuchungen über eindeutige analytische Funktionen. - SpringerVerlag, Berlin, 1968. 